SLAC-PUB-8102

March 1999

\title{
INCLUDING INTERNAL LOSSES IN THE EQUIVALENT CIRCUIT MODEL OF THE SLAC DAMPED DETUNED STRUCTURE (DDS)
}

\author{
R.M. Jones $^{\dagger}$, N.M. Kroll ${ }^{\dagger \dagger}$ and R.H. Miller ${ }^{\dagger}$ \\ $†$ Stanford Linear Accelerator Center, M/S 26, P.O Box 4349, Stanford, CA 94309, USA \\ 屯University of California, San Diego, La Jolla, CA 92093-0319.
}

\begin{abstract}
In the equivalent circuit model for the DDS originally presented no losses were explicitly included in the cell circuits or the manifold circuits. Damping via the manifolds was effected by imposing matching conditions (including the possibility of reflection) on the ends of the manifolds. In this paper we extend the circuit theory to include lossy circuit elements. We discuss and compare shunt conductance and series resistance models for the cells. Manifold damping is modeled by introducing a shunt conductance per unit length in the transmission line elements of the manifolds. We apply the theory to the mitigation of performance degradation associated with fabricationally desirable decoupling of several cells at the ends of the structure from the manifolds.
\end{abstract}

Paper presented at the 1999 Particle Accelerator Conference (PAC99)

New York City, New York, USA

March 29-April 2, 1999

This work is supported by Department of Energy grant numbers DE-AC03-76SF00515 ${ }^{\dagger}$ and DE-FG03-93ER40759. 


\title{
INCLUDING INTERNAL LOSSES IN THE EQUIVALENT CIRCUIT MODEL OF THE SLAC DAMPED DETUNED STRUCTURE (DDS)*
}

\author{
R.M. Jones $^{\dagger}$, N.M. Kroll ${ }^{\dagger \dagger}$ and R.H. Miller ${ }^{\dagger}$ \\ $\uparrow$ Stanford Linear Accelerator Center, M/S 26, P.O Box 4349, Stanford, CA 94309 \\ $\ddagger$ University of California, San Diego, La Jolla, CA 92093-0319.
}

\begin{abstract}
In the equivalent circuit model for the DDS originally presented no losses were explicitly included in the cell circuits or the manifold circuits. Damping via the manifolds was effected by imposing matching conditions (including the possibility of reflection) on the ends of the manifolds. In this paper we extend the circuit theory to include lossy circuit elements. We discuss and compare shunt conductance and series resistance models for the cells. Manifold damping is modeled by introducing a shunt conductance per unit length in the transmission line elements of the manifolds. We apply the theory to the mitigation of performance degradation associated with fabricationally desireable decoupling of several cells at the ends of the structure from the manifolds.
\end{abstract}

\section{INTRODUCTION}

The SLAC DDS acronym refers to detuned accelerator structures in which the transverse wake field is controlled by detuning for proximate bunches and weak damping for remote bunches [1]. The damping is provided by four damping manifolds, waveguide like structures aligned symmetrically along the length of the structure, which drain dipole mode energy into external loads at both ends of the manifolds. In the original equivalent circuit studies, the manifolds were coupled to every structure cell and the manifold loads were matched. Furthermore, the equivalent circuit model [2] included no losses other than those resulting from the external manifold loads. Performance assessments have, however, always taken copper losses into account phenomenologically by multiplying computed wake functions by a decaying exponential with an appropriate damping time characteristic for each structure type, corresponding roughly to a $\mathrm{Q}$ of 6500 at $15 \mathrm{GHz}(181 \mathrm{~ns})$.

Subsequently the effect of manifold mismatch and the effect of decoupling a few cells from the manifolds have received a great deal of attention. Even quite small manifold mismatch leads to significant degradation of the transverse wake function [3,4]. Decoupling a few cells from the manifolds at the downstream end of the structure gives rise to a few modes with relatively high $\mathrm{Q}$ and large kick factors [5], which also leads to significant degradation. It is expected that both of these effects can be ameliorated by the addition of resistive damping to the equivalent circuit model. Adding resistive attenuation to the manifolds is likely to reduce the effect of termination mismatch, while individually damping the downstream cells not connected to the manifold is expected to reduce the $\mathrm{Q}$ of the modes discussed in [5]. In the following sections we first discuss equivalent circuit models which include cell damping and obtain the modified equivalent circuit expressions for the wake impedance defined in [6]. The wake function itself is then obtained by Fourier transforming the wake impedance without employing any direct appeal to contour modification or to analytic properties. This is followed by applications of the theory to uniform cell damping, intended to replace the phenominological model of copper losses mentioned above, and to the effect of damping the downstream cells not connected to the manifold. The effect of manifold attenuation will be discussed in a future publication.

\section{CIRCUIT MODELS OF DAMPING}

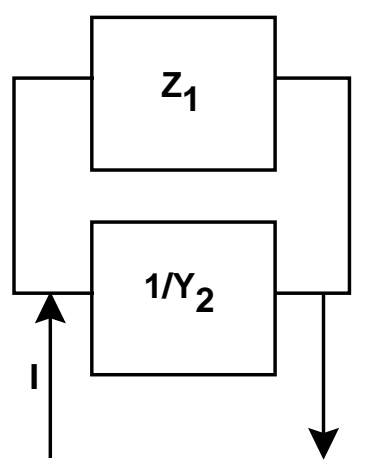

Figure 1: Circuit diagram for including loading

We describe and compare two circuit models of cell damping which we refer to as the series resistance model and the shunt conductance model. In the former, resistances of arbitrary magnitude are inserted in series with the cell loop inductances (see [2], especially Fig. 1), while in the latter, conductances are shunted across the cell loop capacitances. The impedance and inductance in Fig. 1 are given by:

$$
\begin{aligned}
& Z_{1}=j \omega(L+M)+R+Z_{m} \\
& Y_{2}=j \omega C+G
\end{aligned}
$$

where $\mathrm{L}, \mathrm{R}, \mathrm{G}$, and $\mathrm{C}$ are diagonal $2 \mathrm{~N} \times 2 \mathrm{~N}$ matrices $(\mathrm{N}$ being the number of accelerator cells), $\mathrm{M}$ is the mutual 
coupling matrix (it has no diagonal elements), $\mathrm{Z}_{\mathrm{m}}$ is the manifold impedance matrix. Furthermore, all the matrices are symmetric. In the series resistance model $\mathrm{G}=0$ and for the shunt conductance model $\mathrm{R}=0$.

The impedance presented to the terminals may be written:

$$
\begin{gathered}
\mathrm{Z}_{1}=\mathrm{Z}_{1}\left(1+\mathrm{Y}_{2} \mathrm{Z}_{1}\right)^{-1} \\
=\frac{1}{\sqrt{\mathrm{Y}_{2}}} \frac{\sqrt{\mathrm{Y}_{2}} \mathrm{Z}_{1} \sqrt{\mathrm{Y}_{2}}}{1+\sqrt{\mathrm{Y}_{2}} \mathrm{Z}_{1} \sqrt{\mathrm{Y}_{2}}} \frac{1}{\sqrt{\mathrm{Y}_{2}}}
\end{gathered}
$$

We write $Z_{1}$ because the circuit shown in Fig 1 represents the longitudinal impedance. Because $\mathrm{Y}_{2}$ and $\mathrm{Z}_{1}$ do not commute the order of factors in the above eqn. is significant. The second form, with the dimension-less quantity $\sqrt{\mathrm{Y}_{2}} \mathrm{Z}_{1} \sqrt{\mathrm{Y}_{2}}$ is well-suited to expression in terms of our standard scaled variables. The transverse impedance, obtained from the above by application of the Panofsky Wentzel theorem in the usual way leads to an expression analogous to eqn. 5 of [6] with the expression $\overline{\mathrm{H}}\left(1-\mathrm{f}^{2} \overline{\mathrm{H}}\right)^{-1}$ which appears there replaced in the series resistance case by:

$$
\overline{\mathrm{H}}_{\mathrm{Q}}\left(1-\mathrm{f}^{2} \overline{\mathrm{H}}_{\mathrm{Q}}\right)^{-1}
$$

with

$$
\overline{\mathrm{H}}_{\mathrm{Q}}=\overline{\mathrm{H}}-\mathrm{j}\left(\mathrm{f}_{0} \mathrm{fQ}\right)^{-1}, \mathrm{Q}=\mathrm{Q}_{\mathrm{R}}=\omega_{0} \mathrm{~L} / \mathrm{R}=2 \pi \mathrm{f}_{0} \mathrm{~L} / \mathrm{R}
$$

in the shunt resistance case by:

where:

$$
\overline{\mathrm{H}}_{\mathrm{G}}=\mathrm{U}^{-1} \frac{\mathrm{U} \overline{\mathrm{H}} \mathrm{U}}{1-\mathrm{f}^{2} \mathrm{U} \overline{\mathrm{H}} \mathrm{U}} \mathrm{U}^{-1}
$$

$$
\mathrm{U}=\sqrt{1-\mathrm{jf}_{0} /\left(\mathrm{fQ}_{\mathrm{G}}\right)}, \mathrm{Q}_{\mathrm{G}}=\omega_{0} \mathrm{C} / \mathrm{G},
$$

with the approximation

$$
\mathrm{H}_{\mathrm{G}} \approx \frac{1}{2}\left(\overline{\mathrm{H}}\left(1-\mathrm{f}^{2} \overline{\mathrm{H}}_{\mathrm{Q}}\right)^{-1}+\left(1-\mathrm{f}^{2} \overline{\mathrm{H}}_{\mathrm{Q}}\right)^{-1} \overline{\mathrm{H}}\right), \mathrm{Q}=\mathrm{Q}_{\mathrm{G}}
$$

Comparing the series resistance and shunt conductance expressions with equal $Q$ matrices, we see that the denominator matrix is the same in the two cases. For the series case, however, the numerator matrix contains a term $\mathrm{j} /\left(\mathrm{ff}_{0} \mathrm{Q}\right)$ which has a pole at $\mathrm{f}=0$ and gives a non vanishing (and non physical) contribution to the wake function at infinite $s$. In the applications carried out so far this effect of this term has been suppressed be restricting the domain of integration, but in future work the shunt conductance model will be used. We propose to add manifold attenuation to the equivalent circuit model by adding attenuation to the manifold waveguide sections.

\section{EVALUATING THE WAKE FUNCTION}

In reference [6] we computed the (causal) wake function from:

$$
\mathrm{W}_{\mathrm{c}}(\mathrm{s})=\theta(\mathrm{s}) \int_{0}^{\infty} \mathrm{S}(\mathrm{f}) \sin [(2 \pi \mathrm{s} / \mathrm{c}) \mathrm{f}] \mathrm{df}
$$

where $S(f)=-4 \operatorname{Im}\{Z(f-j \varepsilon)\}$. We recall some well known relations to argue that the same expression is appropriate here.

Because $W$ is real, $\operatorname{Re}\{\mathrm{Z}(\mathrm{f}-\mathrm{j} \varepsilon)\}=\operatorname{Re}\{\mathrm{Z}(-\mathrm{f}-\mathrm{j} \varepsilon)\}$, and $\operatorname{Im}\{Z(f-j \varepsilon)\}=-\operatorname{Im}\{Z(-\mathrm{f}-\mathrm{j} \varepsilon)\}$

and thus:

$\mathrm{W}(\mathrm{s})=2 \int_{0}^{\infty} \mathrm{df}\left\{\mathrm{Z}_{\mathrm{r}} \cos [2 \pi \mathrm{s}(\mathrm{f}-\mathrm{j} \varepsilon)]-\mathrm{Z}_{\mathrm{i}} \sin [2 \pi \mathrm{s}(\mathrm{f}-\mathrm{j} \varepsilon)]\right\}$

Define:

$$
\mathrm{W}_{\mathrm{c}}(\mathrm{s})=-4 \theta(\mathrm{s}) \int_{0}^{\infty} \mathrm{dfZ} \sin [2 \pi \mathrm{s}(\mathrm{f}-\mathrm{j} \varepsilon)]
$$

where $\mathrm{Z}=\operatorname{Im}\{\mathrm{Z}\}$ and $\mathrm{Z}_{\mathrm{r}}=\operatorname{Re}\{\mathrm{Z}\}$. If $\mathrm{W}$ vanished for negative $s$ as it is physically required to do, we would have $\mathrm{W}=\mathrm{W}_{\mathrm{c}}$. For then the cos integral must equal minus the sin integral. As has been noted before the equivalent circuit model has a non physical precursor so that the equality of the two terms is only approximate at small $\mathrm{s}$. Physically, however, $\mathrm{W}$ must vanish both for negative $\mathrm{s}$ and at $\mathrm{s}=0$, so we consider $\mathrm{W}_{\mathrm{c}}$ to be a better representation of the wake function than $\mathrm{W}$. While this argument might lead one to conclude that it would be equally justified to use the cos integral, the sin integral is superior both because it vanishes at $\mathrm{s}=0$ and because it is easier to evaluate numerically. Practically speaking infinite integration ranges must always be replaced by finite ones, and generally speaking $\operatorname{Im}\{Z\}$ (in contrast to $\operatorname{Re}\{Z\})$ can be neglected outside the structure pass bands. If one ignores the fact that this argument fails near $f=0$ for the series resistance case and confines the integration to the structure pass bands, the two models should give very similar results.

\section{APPLICATIONS}

The primary impetus of this work was to determine whether the deleterious effect of decoupling the last few downstream cells could be countered by damping these cells and to obtain quantitative information on how much damping would be required. In our previous analysis there were no losses in the circuit model, and we included Ohmic cell losses by including a decay constant after taking the Fourier transform of the spectral function. 


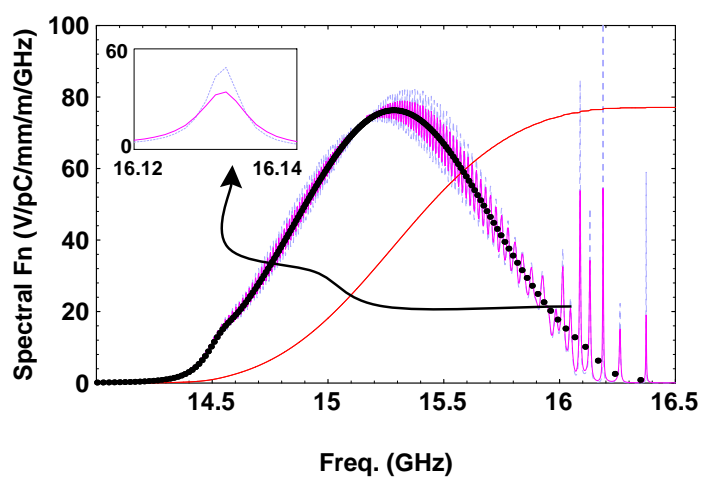

Figure 2: Spectral function for four cells decoupled from the manifold of RDDS1 and all loaded with a cell Q of 6500. Also shown is the smoothed undamped spectral function (indicated by dots).

However, in Fig. 2 the spectral function is calculated including Ohmic cell losses for both the TE and TM mode in all 206 cells. For our previous phenomenological model to have been a faithful representation of damping, the wake computed by both models should be quite similar and, the resulting wake shown in Fig. 3 reveals that the only noticeable difference in the wake is due to a slightly different sampling used in the the two Fourier transforms in the two methods. This gives us confidence in the reliablility of the method and, we calculate the effect of damping the last four and first four cells of the structure with a $\mathrm{Q}$ of 1000 (corresponding to the original design of a manifold-cell Q of 1000), and all other cells have purely copper losses.

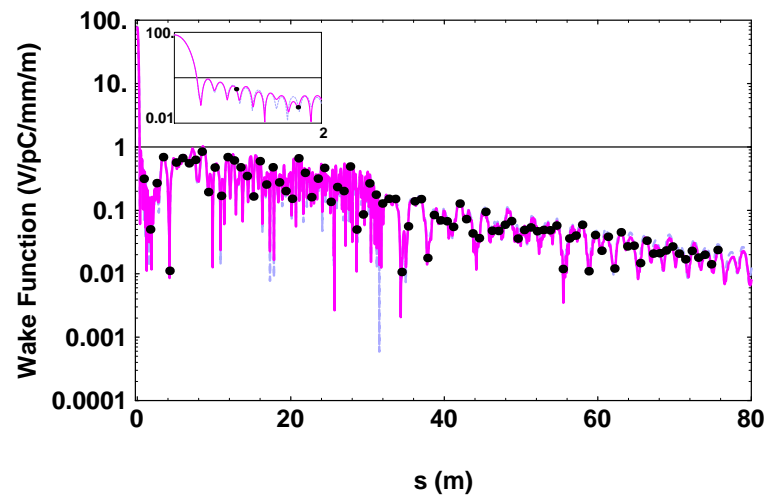

Figure 3: Wake function for four cells decoupled from the manifold of RDDS and all loaded with a cell Q of 6500 , corresponding to all cells being loaded down with copper losses at room temperature. The bunches are spaced at $2.8 \mathrm{~ns}$ and they are indicated by dots.

The spectral function for this situation, shown in Fig 4., has oscillations in the upper frequency end with considerably reduced amplitude compared with those in Fig. 2. Indeed, on computing the modal $\mathrm{Q}$ we find that the $\mathrm{Q}$ for the last 5 resonances ranges from approximately 1200 to 1500 , as compared with a $\mathrm{Q}$ range of 3,000 to 12,800 for the situation in Fig 2. Thus the wakefield, illustrated in Fig. 5, is considerably improved in the region greater than $5 \mathrm{~m}$ or so, in the the neighbourhood of the recoherance peak (about $30 \mathrm{~m}$ or so), and thereafter.

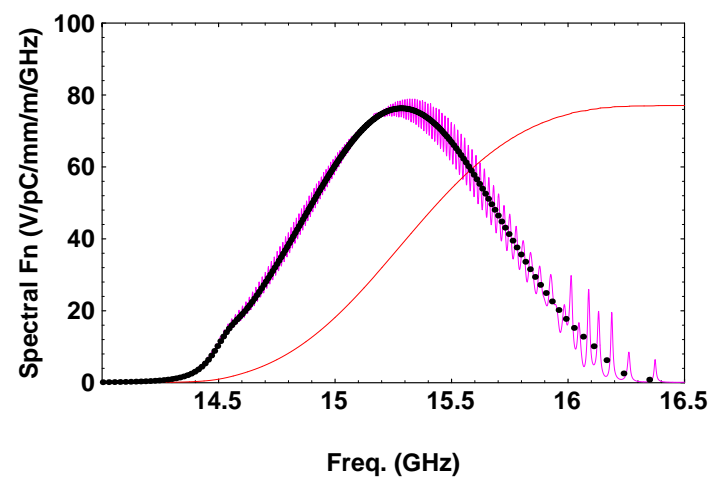

Figure 4: Spectral function for four cells decoupled from the manifold of RDDS and all are loaded with a cell Q of 6500 , apart from the last four and the first four cells which are given $\mathrm{Q}$ of 1000 .

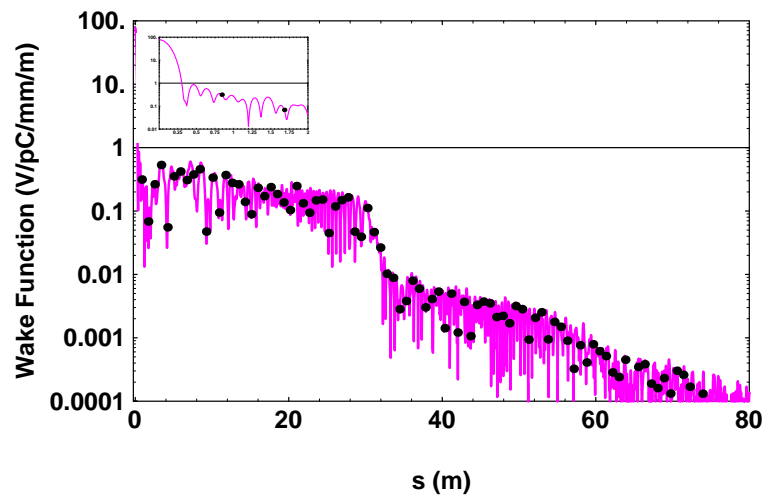

Figure 5: Wake function for four cells decoupled from the manifold of RDDS and all are loaded with a cell Q of 6500 , apart from the last four and the first four cells which are given $\mathrm{Q}$ of 1000 Shown inset is the wakefield for the first $2 \mathrm{~m}$.

\section{ACKNOWLEDGMENTS}

This work is supported by Department of Energy grants DE-FG03-93ER40759 $9^{\ddagger}$ and DE-AC03-76SF00515 ${ }^{\dagger}$.

\section{REFERENCES}

[1] N.M.Kroll, The SLAC Damped Detuned Structure: Concept and Design, 1997 Particle Accelerator Conf. (also SLAC-Pub 7541)

[2] R.M. Jones, K. Ko, N.M. Kroll, R.H, Miller and K.A. Thompson, Equivalent Circuit Analysis of the SLAC Damped Detuned Structure, EPAC96(also SLAC-Pub 7187)

[3] R.H. Miller, et all, A Damped Detuned Structure for the Next Linear Collider, Linac96, (also SLAC-Pub 7288)

[4] R.M. Jones, N.M. Kroll, R.H. Miller, R.D. Miller, and J.W. Wang, Advanced Detuned Development (DDS) at SLAC, Proc. 1997 Particle Accelerator Conf. (also, SLAC-Pub 7537)

[5] R.M. Jones et al, The Transverse Long-Range Wakefield in RDDS1 for the JLC/NLC X-Band Linacs, this conf. FRA37

[6] R.M. Jones., N.M. Kroll and R.H. Miller, A Spectral Function Method Applied To The Calculation Of The SLAC Damped Detuned Structure. Intl. Linac Conf..1996 (SLAC-PUB 7287). 